•综述・

\title{
基于三代测序技术的微生物组学研究进展
}

\author{
许亚昆 ${ }^{1,2}$ 马 越 1,2 胡小茜 ${ }^{1}$ 王 军 $^{1^{*}}$ \\ 1 (中国科学院微生物研究所, 北京 100101) \\ 2 (中国科学院大学, 北京 100049)
}

摘要: 微生物在人类生活中无处不在, 过去人们对微生物的认识仅停留在单菌培养和定性研究上, 而测序技术的 发展极大地促进了微生物组学的研究。越来越多的证据表明: 人体共生微生物、特别是肠道微生物与人类健康息 息相关。二代测序技术凭借其高通量、高准确率和低成本的特点, 成为微生物组学研究中的主流测序技术。但是 随着研究的深入, 二代测序技术的短读长 (< $450 \mathrm{bp}$ )增加了后续数据分析和基因组拼接难度, 也限制了该技术在未 来研究中的应用。在此背景下, 第三代测序技术应运而生。第三代测序技术又称单分子测序, 能够直接对单个DNA 分子进行实时测序, 而不需要经过PCR扩增。第三代测序技术的平均读长在2-10 kb左右, 最高可以达到 $2.2 \mathrm{Mb}$, 实 现了长序列的高通量测序。凭借其超长的测序读长、无GC偏好性等优势, 三代测序技术为微生物基因组全长测序, 组装完整可靠的基因组提供了新的方法。本文在描述三代测序的技术特点和原理的基础上，重点介绍了三代测序 技术在微生物16S/18S rRNA基因测序、单菌的基因组组装以及宏基因组中的研究应用和进展。

关键词：微生物; 三代测序; 16S/18S rRNA; 宏基因组

\section{Analysis of prospective microbiology research using third-generation sequencing technology}

\author{
Yakun $\mathrm{Xu}^{1,2}$, Yue $\mathrm{Ma}^{1,2}$, Xiaoxi $\mathrm{Hu}^{1}$, Jun Wang ${ }^{1}$ \\ 1 Institute of Microbiology, Chinese Academy of Sciences, Beijing 100101 \\ 2 University of Chinese Academy of Sciences, Beijing 100049
}

\begin{abstract}
Microbes are ubiquitous in human life. In years past, the study of microbes has only focused on single-bacteria cultures and qualitative analyses. The development of sequencing technology has greatly enhanced progress in microbiology research and more and more evidence shows that human symbiotic microbes, especially intestinal microbes, are closely related to human health. Second-generation sequencing technology is currently mainstream in microbiology research because of its high throughput, high accuracy and low cost. However, with the deepening complexity of research, the disadvantages of second-generation technology, i.e. short read length $(<450 \mathrm{bp}$ ), lead to subsequent challenges in data analysis and genome assembly, and limit the applicability to future research. In this context, the third-generation sequencing technology comes into being. The third-generation of sequencing (TGS) technology is also called single molecule sequencing. It directly carries out real-time sequencing of single DNA molecules without PCR amplifications. TGS technology significantly increases read length up to $2-10 \mathrm{~kb}$ or even $2.2 \mathrm{Mb}$. Because of its advantages of long read and no preference for GC, TGS provides a new method for full-length gene sequencing that facilitates the assembly of complete and reliable genome maps in microbes and that further reveals the diversity of microbial structures and functions. This review summarizes the technical characteristics and principles of TGS, and then mainly analyzes its applications and progress in 16S/18S rRNA gene sequencing, complete bacterial genome mapping and metagenomics research.
\end{abstract}

Key words: microbes; third-generation sequencing; 16S/18S rRNA; metagenomics

微生物通常指一切难以用肉眼观察到的微小 生物, 包括细菌、病毒、古菌、真菌以及一些微小

收稿日期: 2018-07-30; 接受日期: 2018-12-25

基金项目: 科技部重点研发子课题(2018YFC2000504)和国家自然科学基金(31771481)

* 通讯作者 Author for correspondence. E-mail: junwang@im.ac.cn 
的原生生物。微生物体积微小、结构简单, 却又无 处不在, 在人类健康(Yang \& Tarng, 2018)、工农业 生产(Huang et al, 2018)、环境保护(Liu et al, 2018) 和食品安全(Alori \& Babalola, 2018)等方面都发挥 着重要的作用。微生物传统的研究方法以分离纯菌 培养为主，但由于培养技术的限制，超过 $99 \%$ 的原 核微生物无法在实验室培养得到, 导致微生物结构 和功能多样性的研究一直发展缓慢 (Schloss \& Handelsman, 2005)。

16S/18S rRNA基因存在于所有微生物的基因 组中, 是微生物分类鉴定最常用的标记分子。 Sanger测序技术的兴起(Sanger et al, 1977), 使得 $16 S$ rRNA基因测序在细菌分类学中被广泛应用, 由此发现了大量新的微生物门类, 增加了微生物的 多样性。1998年提出的宏基因组学(Hugenholtz et al, 1998)方法, 是直接从环境样品中获取总的遗传物 质来研究微生物群落的组成和结构, 发现新的功能 基因。宏基因组学的方法不仅解决了微生物分离培 养的难题, 而且可以全面分析微生物群落的多样性 和丰度, 研究微生物之间、微生物和环境或宿主之 间的关系。而在过去的数年间，第三代测序技术的 出现, 以其超长的测序读长、无GC碱基偏好性和速 度快等优势，在以 $16 S / 18 S$ rRNA基因或全基因组为 目标序列的微生物组学研究中取得了一系列进展, 因此微生物的组成和群落结构、代谢特征、系统进 化、微生物与环境的相互作用得到了更深层次的 研究。

\section{测序技术的发展}

\section{1 第一代测序技术}

第一代测序技术(Sanger法)开始于1977年，该 测序方法完成了噬菌体X174全长5,375 个碱基的基 因测序, 由此生命科学研究进入了基因组学时代 (Sanger et al, 1977)。Sanger法即双脱氧终止法(Chain Termination Method), 是指在含有四种脱氧核苷三 磷酸(dNTP)的反应体系中加入一种不同的苂光标 记的双脱氧核苷三磷酸(ddNTP)。由于ddNTP没有 DNA延长所需要的3-OH基团, DNA合成随机地在 G、A、T、C处终止, 然后通过凝胶电泳确定待测 DNA分子的长度。第一代测序技术的测序读长在 1,000-1,500 bp, 但一次只能测一条单独的序列, 导 致测序成本高、通量低, 不能够应用于大规模的基
因测序。

\section{2 第二代测序技术}

二代测序技术又称 “下一代”测序技术 (Next-Generation Sequencing Technology, NGS)。该 技术在保持测序准确度的前提下，主要解决了第一 代测序通量低的问题。二代测序技术可以同时对几 万到几百万条DNA分子进行测定，因此也被称为高 通量测序技术(Metzker, 2010)。二代测序平台主要 有Roche公司的454 (Sogin et al, 2006)、Illumina公司 的Solexa、Hiseq (Gloor et al, 2010)和ABI公司的 Solid等测序仪(Mardis, 2008), 其原理分别为焦磷酸 测序、边合成边测序、连接测序。二代测序技术既 大大降低了测序成本又大幅提高了测序通量，同时 保持了高准确性。比如使用Illumina公司的技术平台, 在 $30 \times$ 测序深度下，完成人类基因组测序仅仅需要 1 周(Niedringhaus et al, 2011)。但二代测序技术的主 要问题在于测序读长短, 只有250-300 bp, 而且由 于测序的系统偏好性，导致有些序列可能被测了很 多次，但也有一些量少的序列无法被大量扩增，造 成信息的丢失。

\section{3 第三代测序技术}

第三代测序技术主要有Pacific Biosciences公司 的SMRT (Single Molecule, Real-Time Sequencing)技 术和Oxford Nanopore公司的Nanopore技术(Eid et al, 2009)。三代测序技术的主要特点是单分子实时测 序、长读长, 但错误率偏高。下面从技术特点和测 序原理两方面来介绍第三代测序技术。

\subsubsection{SMRT技术}

SMRT技术的核心是能够实现单个DNA分子的 测序，并且实时监控测序结果。2013年 Pacific Biosciences公司成功推出商业化的三代测序仪 PacBio RSII后，三代测序开始被广泛应用于微生物 基因组研究中。经过不断的改良和升级, 又在2015 年10月推出全新的PacBio Sequel测序系统, 测序技 术的准确性和通量不断提高。SMRT技术测序系统 主要有 SMRT Cell 、零模波导孔 (Zero-Mode Waveguides, ZMW)和DNA聚合酶。

SMRT Cell是测序芯片, DNA样本完成建库后 装载到Cell中才能上机测序。PacBio RSII系统配套 的一个SMRT Cell的碱基产量为500 M到1 G, 而最 新升级的Sequel系统配套的一个SMRT Cell可产生 5-10 G的数据, 测序通量提升10倍左右。零模波导 
孔ZMW是一种直径为50-100 $\mathrm{nm}$ 的圆形纳米小孔。 当DNA分子进入小孔后, 因为从孔底发出的激发光 不能穿透小孔进入上方的溶液区, 被限制在底部一 个足以覆盖被检测DNA部分的区域, 而共聚焦显微 镜只收集该区域的信号, 从而将背景噪音降到最 低。PacBio RSII使用的一个SMRT Cell中含有 15 万 个ZMW 孔, 而最新的 Sequel 平台配套的升级版 SMRT Cell中含有100万个ZMW孔。DNA聚合酶是 实现SMRT技术长测序读长的关键之一, 它在保持 测序延续性的基础上, 实现了DNA体内的合成速 度。DNA聚合酶能够每秒钟读取10个碱基, 因此测 序速度是化学测序方法的2万倍。

\subsubsection{Nanopore技术}

与以往的测序技术均不同, Nanopore是采用电 信号进行测序(Magi et al, 2017)。2014年, Oxford Nanopore公司开始对外提供Nanopore MinION试用 项目计划, 随后不断对早期版本仪器的高错误率和 低通量问题进行改善。从2016年开始, Nanopore平 台的通量得到较大提升, 错误率也显著降低, 在基 因组中的应用也从微生物小基因组逐渐延伸到复 杂动植物基因组。而更高通量测序平台GridION X5 和PromethION的发布使Nanopore在复杂物种中的 应用更为简单和便捷。新的测序仪在延续MinION 核心测序技术的操作简单和文库制备快等优点的 基础上, 弥补了MinION测序仪通量低以及不适用 于大批量样本或大基因组测序的不足。根据最新的 文献记载, Nanopore MinION测序基于新开发的 BulkVis工具首次生成了单个 $2.2 \mathrm{M}$ 序列(Payne et al, 2018)。

Nanopore测序系统主要包括纳米孔、薄膜和马 达蛋白(motor protein)。纳米孔是一种跨膜蛋白形成 的纳米孔道。不同版本的测序芯片使用不同的跨膜 蛋白，目前R9版的芯片使用的是来自大肠杆菌的 CsgG蛋白质经过基因工程改造后形成的通道。薄膜 是人工合成的具有高电阻的膜, 膜的两侧浸在含有 离子的水溶液中, 通过对膜上施加电势, 离子通过 薄膜上的蛋白纳米孔产生电流。马达蛋白是一种 DNA 解旋酶, 在构建文库时, 马达蛋白和引导接头 (leading adaptor)一同加在DNA分子上, 在测序过程 中, 马达蛋白会对双链DNA解螺旋使其变为单链, 使得单链DNA以一定速度经过纳米孔。

Nanopore技术有3种不同的建库方式: (1)在1D
建库中, 仅有引导接头, 在测序过程中，马达蛋白 对双链DNA解压和解链, 引导接头通过纳米孔, 随 后模板链通过。(2)在2D建库中, 既有引导接头, 还 有连接双链DNA分子的发夹接头(hairpin adaptor)。 在测序过程中，首先是马达蛋白对双链DNA解螺旋 使其变为单链, 引导接头通过纳米孔, 随后模板链 通过, 然后发卡接头和互补链通过。(3)在 $1 \mathrm{D}^{2}$ 建库 中, DNA双链分别通过纳米孔，但并未如2D测序中 通过发卡接头进行连接。当模板链完成测序后, 纳 米孔会捕获互补链的马达蛋白进行互补链测序 (Clarke et al, 2009)。1D测序建库的优势在于文库构 建更便捷，可低至10 min; 相对1D测序，2D测序中 可以得到更长的读长; $1 \mathrm{D}^{2}$ 同时对模板链和互补链 进行测序，可以得到高质量的一致性序列(Jain et al, 2018)。

\section{2 三代测序技术在微生物组学研究中的应月}

\section{$2.116 S / 18 S$ 三代测序}

$16 \mathrm{~S}$ rRNA基因存在于所有原核微生物的基因 组中, 序列长度约为 $1,500 \mathrm{bp}$ (18S rRNA基因存在 于所有真核微生物的基因组中，序列全长为 1,500-2,000 bp)。16S rRNA 基因有 9 个高变区 (V1-V9), 在一定程度上反映了微生物间的进化差 异，可以利用高变区对不同菌属、菌种的细菌进行 分类鉴定; 而与高变区间隔分布的 8 个高度保守区 域在细菌漫长的进化过程中则保持了结构和功能 的稳定性。1977年, Woese等依据细菌16S rRNA基因 序列上的区别, 提出了三域系统, 并分别命名为细 菌域、古菌域和真核域(Woese \& Fox, 1977)。目前 16S/18S rRNA基因是研究微生物系统发育和分类 鉴定最常用的分子标记(Woese, 1987)。

基于 $16 \mathrm{~S} / 18 \mathrm{~S}$ 的微生物基因测序即是利用 16S/18S rRNA保守区域的基因序列设计引物，扩增 研究所需的基因片段(Jonasson \& Monstein, 2002)。 包括基因组DNA的获取; 设计标准引物对16S/18S 基因的一个或多个区进行扩增; 聚类，按照一定的 相似性将扩增得到的序列进行分组，细菌中的分类 操作单元(operational taxonomic unit, OTU)是基于 序列 $97 \%$ 相似性的分类; 然后通过数据库比对为 OTU的代表性序列提供分类学注释。

三代测序技术由于超长的测序读长，在微生物 的分类鉴定和群落多样性的研究中更具有优势。二 
(a)

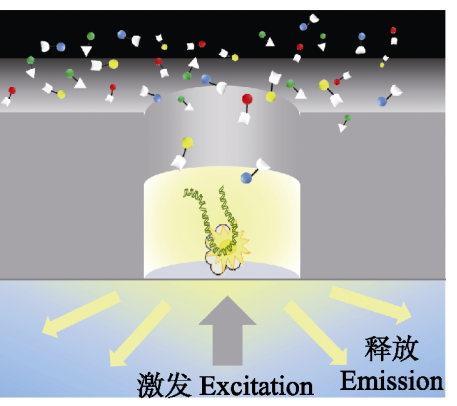

(b)

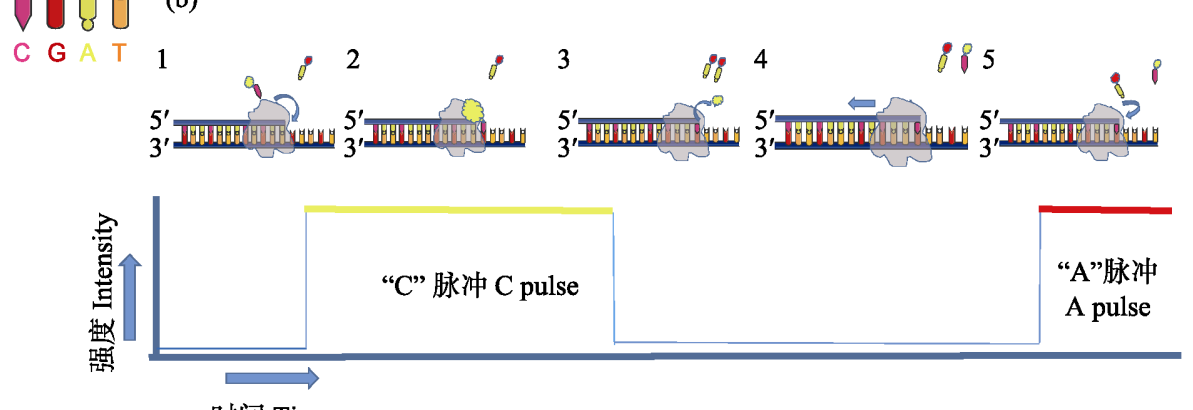

时间 Time

图1 PacBio SMRT测序原理。(a)在零模波导孔(Zero-Mode Waveguides, ZMW)中，单个DNA分子模板与引物和聚合酶结合 后, 被固定到ZMW孔底部。DNA合成开始时, 新加入的荧光标记的dNTP由于碱基配对在ZMW底部停留较长时间, 激发后 发出对应的荧光信号被共聚焦显微镜实时记录; (b) 1)荧光标记胞嘧啶脱氧核苷酸; 2)胞嘧啶脱氧核苷酸进入DNA链配对, 发 射荧光信号; 3)荧光基团被DNA聚合酶切除, 荧光消失; 4)标记新的脱氧核苷酸; 5)继续新一轮合成。

Fig. 1 Schematic diagram of PacBio single molecule real-time sequencing. (a) In the ZMW hole, a single DNA molecule template combined with primers and polymerase is bind to the bottom of ZMW hole. At the beginning of DNA sequencing, the newly added fluorescent labeled dNTP remained at the bottom of ZMW for a long time due to base pairing, and the corresponding fluorescent signals were recorded by confocal microscopy in real time. (b) (1) Fluorescence labeling cytosine deoxynucleotides; (2) Cytosine deoxynucleotides entering DNA chain pairing, emitting fluorescent signals; (3) Fluorescent group is removed by DNA polymerase, fluorescence disappeared; (4) Label new deoxynucleotides; (5) Continue a new round.

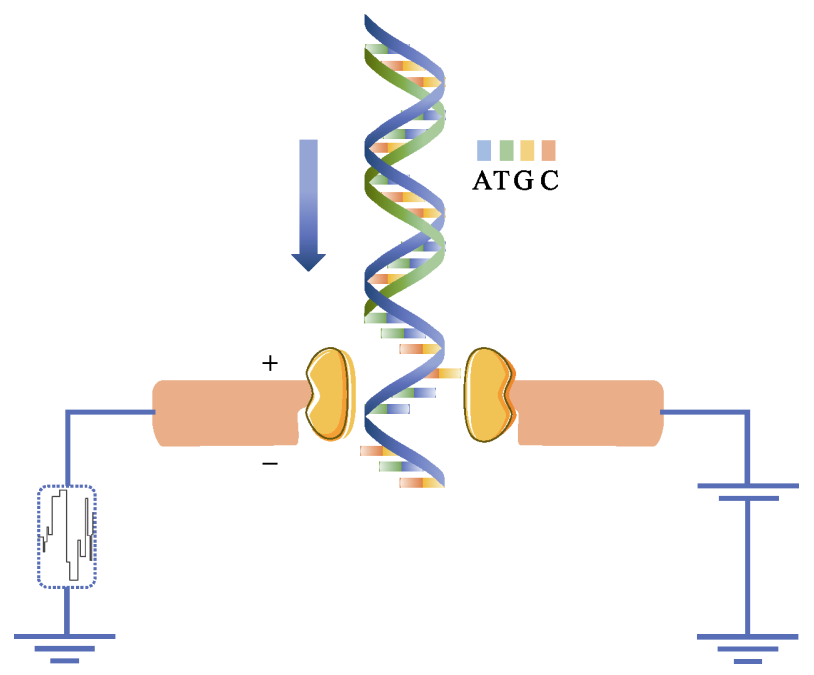

图2 Nanopore利用电信号检测出DNA的碱基序列。纳米孔 直径很小, 仅仅允许单个核苷酸通过。当DNA单链通过的时 候, 就会对离子的流动造成阻碍, 从而使流过纳米孔的电流 强度发生变化。由于ATCG四种碱基的带电性质不一样, 造 成电流大小的波动也不一样, 因此可根据电流的变化鉴定 所通过的碱基类型。

Fig. 2 Nanopore DNA sequencing using electronic signals as detection methods. The diameter of the nanoscale is very small that only a single DNA molecule is allowed to pass through. When a single strand of DNA passes through, it blocks the flow of ions and changes the current intensity across the nanopore. Because the charge properties of the four bases of ATCG are different, the type of base passed is identified according to the change of current.

代测序通常只能获得1-2段高变区域，而三代测序 的读长提升了 $2-3$ 倍以上, 因而也提升了物种鉴定
的分辨率。研究人员对同一人的肠道菌群样本分别 进行二代和三代的16S rRNA基因测序, 其中使用 Illumina Miseq平台对16S rRNA基因的V3-V4区进 行扩增测序，使用PacBio SMRT技术直接读取和覆 盖16S rRNA基因的V3-V7区，实验结果表明有更 多的OTUs基于SMRT测序数据被鉴定出来(Franzen et al, 2015)。Cusco等(2018)将Nanopore MinION应用 于16S rRNA基因测序，在犬类的皮肤微生物群体 中发现了新的细菌门类。在另外一项针对已知菌种 混合组成的模拟菌群的研究中, Nanopore MinION 的测序数据使得一些物种能鉴定到种的水平 (Benitez-Paez et al, 2016)。因此当三代测序技术的读 长可以覆盖到更多基因片段时, 测序结果能够更准 确地检测到微生物群体多样性组成。

\section{2 细菌/真菌基因组三代测序}

在微生物组学的研究中, 已有参考基因组的微 生物数量远远低于自然界存在的微生物数量, 除了 微生物难以纯化培养外, 还有一部分原因是二代测 序读长短, 难以解决细菌/真菌基因组中的高重复 区域和高GC区域的组装问题，因此组装后的基因 组中往往存在很多gap。而三代测序技术以其超长 的测序读长和无 GC 偏好性克服了以上部分难题, 在单细菌/真菌的基因组组装中取得了很大突破。

2013年Chin等就使用SMRT技术组装出 16 个微 生物基因组，与参考基因组一致性达 $99.9999 \%$ 
表1 三代测序技术的比较

Table 1 Comparison of three generation sequencing technologies

\begin{tabular}{|c|c|c|c|c|c|}
\hline & $\begin{array}{l}\text { 技术平台 } \\
\text { Technical } \\
\text { platform }\end{array}$ & $\begin{array}{l}\text { 测序原理 } \\
\text { Principle of } \\
\text { sequencing }\end{array}$ & $\begin{array}{l}\text { 测序读长 } \\
\text { Read length }\end{array}$ & $\begin{array}{l}\text { 优点 } \\
\text { Advantages }\end{array}$ & $\begin{array}{l}\text { 缺点 } \\
\text { Limitations }\end{array}$ \\
\hline $\begin{array}{l}\text { 第一代 } \\
\text { The first } \\
\text { generation }\end{array}$ & Sanger & $\begin{array}{l}\text { 可中断测序 } \\
\text { Chain-terminating } \\
\text { sequencing }\end{array}$ & $600-1,000$ bp & $\begin{array}{l}\text { 读长长; 准确率高; 能很好地 } \\
\text { 处理一些重复序列和多聚序列 } \\
\text { Long reads; high accuracy; } \\
\text { good ability to deal with } \\
\text { repetitive and homopolymer }\end{array}$ & $\begin{array}{l}\text { 通量低; 样品制备成本高, } \\
\text { 难以做大量的平行测序 } \\
\text { Low throughput; high cost of Sanger } \\
\text { sample preparation; making massively } \\
\text { parallel sequencing prohibitive. }\end{array}$ \\
\hline
\end{tabular}

\begin{tabular}{|c|c|c|c|c|c|}
\hline \multirow[t]{3}{*}{$\begin{array}{l}\text { 第二代 } \\
\text { The second } \\
\text { generation }\end{array}$} & Roche/454 & $\begin{array}{l}\text { 焦磷酸测序 } \\
\text { Pyrosequencing }\end{array}$ & 200-400 bp & $\begin{array}{l}\text { 在二代测序中读长最长; 高通量 } \\
\text { Longest read lengths among the } \\
\text { second-generation; high } \\
\text { throughput. }\end{array}$ & $\begin{array}{l}\text { 样品制备较难; 难于处理重复和 } \\
\text { 同种碱基多聚区域 } \\
\text { Challenging sample preparation; } \\
\text { hard to deal with repetitive/homopo- } \\
\text { lymer regions. }\end{array}$ \\
\hline & Illumina & $\begin{array}{l}\text { 边合成边测序 } \\
\text { Sequencing by synthesis }\end{array}$ & $2 \times 150$ bp & $\begin{array}{l}\text { 高通量 } \\
\text { Very high throughput }\end{array}$ & $\begin{array}{l}\text { 读长短 } \\
\text { Short reads }\end{array}$ \\
\hline & ABI/Solid & $\begin{array}{l}\text { 连接测序 } \\
\text { Sequencing by } \\
\text { ligation }\end{array}$ & 25-35 bp & $\begin{array}{l}\text { 高通量; 成本低 } \\
\text { High throughput; low cost. }\end{array}$ & $\begin{array}{l}\text { 测序运行时间长; 读长短, 造成后续 } \\
\text { 的数据分析困难和基因组拼接困难 } \\
\text { Long sequencing runs (days); short } \\
\text { reads, resulting in difficulties in } \\
\text { subsequence data analysis and genome } \\
\text { assembly. }\end{array}$ \\
\hline \multirow[t]{2}{*}{$\begin{array}{l}\text { 第三代 } \\
\text { The third } \\
\text { generation }\end{array}$} & PacBio SMRT & $\begin{array}{l}\text { 边合成边测序/ } \\
\text { DNA聚合酶 } \\
\text { Sequencing by } \\
\text { synthesis/DNA } \\
\text { polymerase }\end{array}$ & $\sim 1,000$ bp & $\begin{array}{l}\text { 高平均读长; 不需要扩增; } \\
\text { 最长单个读长接近 } 100 \mathrm{~kb} \\
\text { Long average read length; } \\
\text { no amplification of sequencing } \\
\text { fragments; longest individual } \\
\text { reads approach } 100 \mathrm{~kb} \text {. }\end{array}$ & $\begin{array}{l}\text { 错误率高; 依赖DNA聚合酶的活性 } \\
\text { Low accuracy; dependence on DNA } \\
\text { polymerase activity. }\end{array}$ \\
\hline & Nanopore & $\begin{array}{l}\text { 电信号测序/ } \\
\text { 核酸外切酶 } \\
\text { Electronic signals } \\
\text { sequencing/exonuclease }\end{array}$ & $\begin{array}{l}\text { 最大记载2.2 M } \\
\text { Maximum } \\
\text { record } 2.2 \mathrm{M}\end{array}$ & $\begin{array}{l}\text { 读长超长; 电学测序; 方便携带 } \\
\text { Over-long read; electronic } \\
\text { sequencing; portable. }\end{array}$ & $\begin{array}{l}\text { 错误率高 } \\
\text { High sequencing error }\end{array}$ \\
\hline
\end{tabular}

(Chin et al, 2013)。Brown等(2014)分别使用Illumina 和454这些二代测序平台和PacBio RS II平台技术对 梭状芽狍杆菌(Clostridium autoethanogenum)进行基 因组装, 发现二代测序平台组装得到Clostridium的 基因组仍然存在一些gap, 而使用PacBio SMRT测 序直接组装得到一条完整的Contig; 并且之前由于 技术的限制，无法区分 C. autoethanogenum 和 C. ljungdahlii两支菌株，而基于PacBio SMRT的全基 因组测序, 发现两者在CRISPR系统、氢化酶等方面 具有显著差异。Ludden等(2017)用Nanopore MinION 测序平台首次组装出了Enterobacter kobei的基因组 完成图, 并且组装出编码blaOXA-48基因的质粒。

Wick等(2017)对12个不同种的克雷伯氏肺炎菌进行 测序, 然后与前期二代数据混合组装, 既保证了测 序数据的完整性, 又保证了测序的准确性, 最终都 组装成了基因组完成图。此外假单胞菌Pseudomonas koreensis P19E3的基因组含有长达70 kb的重
复序列, 使用二代平台的测序数据无法组装到完整 的基因组, 而Schmid (2018)使用Nanopore MinION 测序仪, 首次完成了对 Pseudomonas koreensis P19E3的基因组组装。

真菌基因组大小在2.5-150 Mb之间, 介于细菌 和大型动植物之间, 随着三代测序平台的改进和升 级, 测序通量的不断提升, 三代测序技术在基因组 研究中的应用也逐渐从细菌等小型基因组延伸到 真菌等大型基因组中。Orpinomyces sp. strain C1A 菌株是一种寄生在大型家畜肠胃中的厌氧真菌, 基 因组超过 $100 \mathrm{Mb}, \mathrm{GC}$ 含量只有 $17 \%$, 是目前已知的 GC含量最低的物种之一。Yousse等(2013)在对该菌 株的基因组组装中发现, 对比 Illumina测序数据得 到的组装结果, PacBio RS的测序数据组装得到的 contigs更长、数量大幅降低, 而且发现 $\mathrm{C} 1 \mathrm{~A}$ 菌株具 有降解木质素的能力。此外Faino等(2015)基于 PacBio RS II平台对黄萎病菌菌株JR2和VdLs17测 
序并组装, 最终都得到了8 条完整的染色体。2017 年荷兰研究者Jansen等分离出了 DDNA\#1酵母菌 株DNA，使用 Illumina和 Nanopore MinION分别 进行测序，通过Illumina测序数据组装得到了 14,764 contigs，而使用 Nanopore测序数据组装 得到的contigs仅 61条，大幅度提高了酵母菌基 因组数据的质量和完整度(Liem et al, 2017)。

\section{3 宏基因组三代测序}

宏基因组学是将环境中的微生物群落作为整 体进行研究，与 $16 S / 18 S$ 或全基因组分析相比，宏基 因学能够对微生物群体基因组成及其功能、微生物 群体的多样性、微生物与环境、微生物与宿主之间 的关系进行全面解读。2016年Hug等就利用宏基因 组技术发现了 1,000 多种未被培养或者了解甚少的 微生物体, 进一步扩展了微生物的多样性, 而新的 细菌门的出现, 也重新补充了进化树三域系统(Hug et al, 2016)。Fierer等(2007)通过构建牧场、沙漠、 雨林土壤宏基因组文库, 对环境中细菌、古生菌、 真菌及病毒多样性进行了研究。结果发现土壤环境 中包含着大量不可培养的新病毒种类, 其基因型特 征与常规培养获得的病毒具有很大的差异。除此之 外, 宏基因组学在海洋微生物多样性的探索中也取 得了很大的进展，促进了海洋微生物资源的开发。 2015年Sunagawa领导的团队分析了来自世界各地 的 68 个地点的远洋中上层水域的共 243 个样本, 构 建了含有 $4 \times 10^{7}$ 个非㝋余基因的海洋微生物宏基因 组库, 并提出在垂直水平上海洋微生物群落的变化 主要是由温度决定的; 同时对139个富含原核微生 物的样本进行统计分析, 共发现了5,755 个同源微 生物组, 其中有 $40 \%$ 的同源组与人类肠道菌群的同 源组相同(Sunagawa et al, 2015)。

二代测序应用于宏基因组学时, 由于读长过短 会导致一些基因信息的丢失。相比之下，宏基因组 学三代测序结果可以更加真实地反映菌群的组成 情况, 较为准确地挖掘出新的功能基因。例如研究 人员使用二代和三代测序平台分别对同一微生物 发酵池样品进行了宏基因组测序分析, 并对结果做 了比较。发现基于Illumina测序数据的组装, 由于读 长较短, 结果存在大量不同物种间的同源序列错误 组装, 无法真实呈现菌群组成; 而PacBio RS II得到 的结果真实反映了两种优势菌株的组成情况(Frank et al, 2016)。Tsai等(2016)也采用了PacBio RS II和
Illumina HiSeq两种测序平台对人类手部和足部的 菌群进行宏基因组分析，结果表明三代测序技术显 著减少了 contigs的数量, 大大降低了序列拼接和基 因组组装的难度, 而且从人的皮肤菌群样本中组 装、注释、构建获得一例未知微生物的高质量基因 组。在对Sakinaw Lake水体微生物的研究中, Singer 等(2016)发现SMRT和Illumina的测序数据在门的水 平上没有太大差别, 但是随着群落复杂度的增加, 二代和三代的测序数据在群落结构和系统发育分 辨率上显示出显著差异。

新型三代测序设备的出现还扩展了宏基因组 研究的应用场景。目前基因测序的工作主要局限于 实验室，而Nanopore MinION这种便携式测序仪为 开展基因组实时测序提供了便利，在鉴定新的病原 体和细菌基因组测序方面得到了广泛应用。例如猪 的肠道疾病通常是由不同的病原体引起的，宏基因 组学是一种常用的诊断方法, 而使用Nanopore技术 在3小时内就可以完成样品测序和致病菌的诊断 (Theuns et al, 2018)。MinION测序仪对于样本的预 处理要求也较低, 对一些高致病性病毒如奇昆古尼 亚病毒、埃博拉病毒和丙型乙肝病毒, MinION测序 仪可以直接使用临床样本进行检测, 而不需要病毒 的纯化培养(Greninger et al, 2015)。在埃博拉等暴发 疾病的实时检测中，研究人员就利用这种便携式测 序仪在整个基因组库中鉴别出了新型的单核苷酸 多态性(SNPs), 为流行病暴发的预警和病原体的进 化提供了重要的方法(Quick et al, 2016)。

目前RNA病毒的基因组研究通常是先反转录 为cDNA再进行测序。最近美国的研究者通过设计 一种针对流感病毒基因组的Nanopore测序接头RTA, 实现了对流感病毒 RNA 的直接测序 (Callaway, 2018)。而且传统方法对表观遗传修饰的分析, 需要 对样本进行亚硫酸盐处理或者抗体沉降(pull-down) 的预处理，增加了检测的难度。通过Nanopore技术， Andrew Smith研究团队直接对大肠杆菌全长16S核 糖体RNA进行测序, 从采样到出结果只需要2小时。 通过数据分析, 该研究小组在 $16 S$ rRNA的已知位 点鉴定出了 7-甲基鸟苷(m7G), 并且观察到了假尿 嘧啶核苷等在内的表观遗传修饰的存在(Smith et al, 2017)。中国科学院水生生物研究所赵亮博士通过 PacBio RS II测序, 获得3个微囊藻菌株的全基因组 甲基化修饰图谱，发现了18个未被鉴定的甲基化模 
块, 也发现不同微囊藻菌株的甲基化和甲基转移酶 含量差异很大(Zhao, 2018)。

三代测序技术的兴起, 极大地促进了微生物组 学的发展, 但是错误率偏高是三代测序技术面临的 最大问题。15\%-40\%的错误率，大大地高于二代测 序技术 $(<1 \%)$, 成为限制其商业应用开展的重要原 因。不过三代测序的错误是随机发生的, 可以靠覆 盖度来纠错(但这又要增加测序成本)。为了提升准 确率, PacBio公司对SMRT技术配套的技术不断优 化, 文献中经常看到的P4C2、P5C3以及P6C4代表的 就是不断升级的试剂。同时, PacBio独有的环形一致 性测序模式(circular-consensus sequence, CCS)极大 地提高了单碱基测序的准确率。Nanopore公司也不 断从Flow Cell、纳米孔、测序试剂和信号捕获及碱 基识别软件等方面进行升级改进。其中, 纳米孔最 开始为R6, 后来不断升级而出现了 R7, R8, R9版本, 到现在已经升级为R9.4, 随之而来的是准确率和通 量的提升。而且目前已经有比较成熟的软件Canu专 门处理三代技术这类错误率较高的测序数据。Canu 采用了先纠错、修整再组装的策略, 通过序列与序 列之间的重叠, 进行单碱基的错误纠正, 测序覆盖 度越高, 准确率就越高。

\section{3 结语}

近年来, 微生物组学是生命科学研究的热门领 域, 而微生物组的深入研究和理解得益于测序技术 的快速发展。第三代测序技术的出现，实现了很多 技术上的突破：(1)凭借其超长读长的优势，解决了 二代测序技术读长过短的问题，在微生物基因组学 研究中减少了后续的基因组组装和注释的工作量, 节省了大量的时间; (2) SMRT技术利用DNA聚合酶 自身的合成速度, 配上高分辨率的光学检测系统, 实现了实时快速检测; (3)三代单分子测序技术的发 展也让通过测序实时读取DNA碱基修饰成为可能, 这对在基因组水平直接研究表观遗传现象有极大 的帮助; (4) Nanopore技术还可以直接对RNA进行 测序, 省去了反转录过程。

但由于三代测序较高的错误率, 目前三代测序 技术在微生物组中的研究往往需要与二代测序数 据结合使用, 即利用二代测序数据对三代测序数据 进行校正, 也就意味着在实验中至少要制备 2 个不 同的文库，造成了一定的人力和物力成本的增加。
随着三代技术的不断改进和新的组装方法的出现， 测序的准确性和通量也在逐步上升。相信在不久的 将来，基于三代测序技术的微生物组学研究能够从 获得高质量的基因组装结果，逐渐转向对物种生物 学特征和进化历程的深入研究, 研究策略也由单一 组学测序逐渐延伸为基因组、转录组、代谢组和表 观遗传组的多组学分析。

\section{参考文献}

Alori ET, Babalola OO (2018) Microbial inoculants for improving crop quality and human health in Africa. Frontiers in Microbiology, 9, 2213.

Benitez-Paez A, Portune KJ, Sanz Y (2016) Species-level resolution of 16S rRNA gene amplicons sequenced through the MinION $^{\mathrm{TM}}$ portable nanopore sequencer. GigaScience, $5,4$.

Brown SD, Nagaraju S, Utturkar S, De Tissera S, Segovia S, Mitchell W, Land ML, Dassanayake A, Kopke M (2014) Comparison of single-molecule sequencing and hybrid approaches for finishing the genome of Clostridium autoethanogenum and analysis of CRISPR systems in industrial relevant Clostridia. Biotechnology for Biofuels, 7, 40.

Callaway E (2018) Flu virus finally sequenced in its native form. Nature, 556, 420.

Chin CS, Alexander DH, Marks P, Klammer AA, Drake J, Heiner C, Clum A, Copeland A, Huddleston J, Eichler EE, Turner SW, Korlach J (2013) Nonhybrid, finished microbial genome assemblies from long-read SMRT sequencing data. Nature Methods, 10, 563-569.

Clarke J, Wu HC, Jayasinghe L, Patel A, Reid S, Bayley H (2009) Continuous base identification for single-molecule nanopore DNA sequencing. Nature Nanotechnology, 4, 265-270.

Cusco A, Vines J, D’Andreano S, Riva F, Casellas J, Sánchez A, Francino O (2018) Using MinION to characterize dog skin microbiota through full-length 16S rRNA gene sequencing approach. bioRxiv, doi: https://doi.org/10.1101/167015.

Eid J, Fehr A, Gray J, Luong K, Lyle J, Otto G, Peluso P, Rank D, Baybayan P, Bettman B, Bibillo A, Bjornson K, Chaudhuri B, Christians F, Cicero R, Clark S, Dalal R, Dewinter A, Dixon J, Foquet M, Gaertner A, Hardenbol P, Heiner C, Hester K, Holden D, Kearns G, Kong XX, Kuse R, Lacroix Y, Lin S, Lundquist P, Ma CC, Marks P, Maxham M, Murphy D, Park I, Pham T, Phillips M, Roy J, Sebra R, Shen G, Sorenson J, Tomaney A, Travers K, Trulson M, Vieceli J, Wegener J, Wu D, Yang A, Zaccarin D, Zhao P, Zhong F, Korlach J, Turner S (2009) Real-time DNA sequencing from single polymerase molecules. Science, 323, 133-138.

Faino L, Seidl MF, Datema E, van den Berg GC, Janssen A, Wittenberg AH, Thomma BP (2015) Single-molecule 
real-time sequencing combined with optical mapping yields completely finished fungal genome. mBio, 6, e00936-15.

Fierer N, Breitbart M, Nulton J, Salamon P, Lozupone C, Jones R, Robeson M, Edwards RA, Felts B, Rayhawk S, Knight R, Rohwer F, Jackson RB (2007) Metagenomic and small-subunit rRNA analyses reveal the genetic diversity of bacteria, archaea, fungi, and viruses in soil. Applied and Environmental Microbiology, 73, 7059-7066.

Frank JA, Pan Y, Tooming-Klunderud A, Eijsink VGH, McHardy AC, Nederbragt AJ, Pope PB (2016) Improved metagenome assemblies and taxonomic binning using long-read circular consensus sequence data. Scientific Reports, 6, 25373

Franzen O, Hu J, Bao X, Itzkowitz SH, Peter I, Bashir A (2015) Improved OTU-picking using long-read 16S rRNA gene amplicon sequencing and generic hierarchical clustering. Microbiome, 3, 43.

Gloor GB, Hummelen R, Macklaim JM, Dickson RJ, Fernandes AD, MacPhee R, Reid G (2010) Microbiome profiling by Illumina sequencing of combinatorial sequence-tagged PCR products. PLoS ONE, 5, e15406.

Greninger AL, Naccache SN, Federman S, Yu GX, Mbala P, Bres V, Stryke D, Bouquet J, Somasekar S, Linnen JM, Dodd R, Mulembakani P, Schneider BS, Muyembe-Tamfum JJ, Stramer SL, Chiu CY (2015) Rapid metagenomic identification of viral pathogens in clinical samples by real-time nanopore sequencing analysis. Genome Medicine, 7, 99.

Huang ZR, Hong JL, Xu JX, Li L, Guo WL, Pan YY, Chen SJ, Bai WD, Rao PF, Ni L, Zhao LN, Liu B, Lv XC (2018) Exploring core functional microbiota responsible for the production of volatile flavour during the traditional brewing of Wuyi Hong Qu glutinous rice wine. Food Microbiology, 76, 487-496.

Hug LA, Baker BJ, Anantharaman K, Brown CT, Probst AJ, Castelle CJ, Butterfield CN, Hernsdorf AW, Amano Y, Ise K, Suzuki Y, Dudek N, Relman DA, Finstad KM, Amundson R, Thomas BC, Banfield JF (2016) A new view of the tree of life. Nature Microbiology, 1, 16048.

Hugenholtz P, Pitulle C, Hershberger KL, Pace NR (1998) Novel division level bacterial diversity in a Yellowstone hot spring. Journal of Bacteriology, 180, 366-376.

Jain M, Koren S, Miga KH (2018) Nanopore sequencing and assembly of a human genome with ultra-long reads. Nature Biotechnology, 36, 338-345.

Jonasson J, Monstein HJ (2002) Classification, identification and subtyping of bacteria based on pyrosequencing and signature matching of $16 \mathrm{~s}$ rDNA fragments. Apmis, 110, 263-272.

Liem M, Jansen HJ, Dirks RP, Henkel CV, van Heusden GPH, Lemmers R, Omer T, Shao S, Punt PJ, Spaink HP (2017) De novo whole-genome assembly of a wild type yeast isolate using nanopore sequencing. F1000Research, 6, 618.
Liu H, Hu Z, Zhang Y, Zhang J, Xie H, Liang S (2018) Microbial nitrogen removal of ammonia wastewater in poly (butylenes succinate)-based constructed wetland: Effect of dissolved oxygen. Applied Microbiology and Biotechnology, 102, 9389-9398.

Ludden C, Reuter S, Judge K, Gouliouris T, Blane B, Coll F, Naydenova P, Hunt M, Tracey A, Hopkins KL, Brown NM, Woodford N, Parkhill J, Peacock SJ (2017) Sharing of carbapenemase-encoding plasmids between Enterobacteriaceae in UK sewage uncovered by MinION sequencing. Microbial Genomics, 3, 1-12.

Magi A, Semeraro R, Mingrino A, Giusti B, D’Aurizio R (2017) Nanopore sequencing data analysis: State of the art, applications and challenges. Briefings in Bioinformatics, 19, 1256-1272.

Mardis ER (2008) Next-generation DNA sequencing methods. Annual Review of Genomics and Human Genetics, 9, 387-402.

Metzker ML (2010) Sequencing technologies-The next generation. Nature Reviews Genetics, 11, 31-46.

Niedringhaus TP, Milanova D, Kerby MB, Snyder MP, Barron AE (2011) Landscape of next-generation squencing technologies. Analytical Chemistry, 83, 4327-4341.

Payne A, Holmes N, Rakyan V, Loose M (2018) Whale watching with BulkVis: A graphical viewer for Oxford Nanopore bulk fast5 files. bioRxiv, doi: https://doi.org/10.1101/ 312256.

Quick J, Loman NJ, Duraffour S, Simpson JT, Ettore S, Cowley L, Bore JA, Koundouno R, Dudas G, Mikhail A, Ouedraogo N, Afrough B, Bah A, Baum JHJ, Becker-Ziaja B, Boettcher JP, Cabeza-Cabrerizo M, Camino-Sanchez A, Carter LL, Doerrbecker J, Enkirch T, Garcia-Dorival I, Hetzelt N, Hinzmann J, Holm T, Kafetzopoulou LE, Koropogui M, Kosgey A, Kuisma E, Logue CH, Mazzarelli A, Meisel S, Mertens M, Michel J, Ngabo D, Nitzsche K, Pallasch E, Patrono LV, Portmann J, Repits JG, Rickett NY, Sachse A, Singethan K, Vitoriano I, Emanaberhan RLY, Zekeng EG, Racine T, Bello A, Sall AA, Faye O, Faye O, Magassouba N, Williams CV, Amburgey V, Winona L, Davis E, Gerlach J, Washington F, Monteil V, Jourdain M, Bererd M, Camara A, Somlare H, Camara A, Gerard M, Bado G, Baillet B, Delaune D, Nebie KY, Diarra A, Savane Y, Pallawo RB, Gutierrez GJ, Milhano N, Roger I, Williams CJ, Yattara F, Lewandowski K, Taylor J, Rachwal P, Turner DJ, Pollakis G, Hiscox JA, Matthews DA, O’Shea MK, Johnston AM, Wilson D, Hutley E, Smit E, DiCaro A, Wolfel R, Stoecker K, Fleischmann E, Gabriel M, Weller SA, Koivogui L, Diallo B, Keita S, Rambaut A, Formenty P, Gunther S, Carroll MW (2016) Real-time, portable genome sequencing for Ebola surveillance. Nature, 530, 228-232.

Sanger F, Nicklen S, Coulson AR (1977) DNA sequencing with chain-terminating inhibitors. Proceedings of the National Academy of Sciences, USA, 74, 5463-5467. 
Schloss PD, Handelsman J (2005) Metagenomics for studying unculturable microorganisms: Cutting the Gordian knot. Genome Biology, 6, 229.

Schmid M, Frei D, Patrignani A, Schlapbach R, Frey JE, Remus-Emsermann MNP, Ahrens CH (2018) Pushing the limits of de novo genome assembly for complex prokaryotic genomes harboring very long, near identical repeats. Nucleic Acids Research, 46, 8953-8965.

Singer E, Bushnell B, Coleman-Derr D, Bowman B, Bowers RM, Levy A, Gies EA, Cheng JF, Copeland A, Klenk HP, Hallam SJ, Hugenholtz P, Tringe SG, Woyke T (2016) High-resolution phylogenetic microbial community profiling. ISME Journal, 10, 2020-2032.

Smith AM, Jain M, Mulroney L, Garalde DR, Akeson M (2017) Reading canonical and modified nucleotides in 16S ribosomal RNA using nanopore direct RNA sequencing. bioRxiv, doi: https://doi.org/10.1101/132274.

Sogin ML, Morrison HG, Huber JA, Mark Welch D, Huse SM, Neal PR, Arrieta JM, Herndl GJ (2006) Microbial diversity in the deep sea and the underexplored "rare biosphere". Proceedings of the National Academy of Sciences, USA, 103, 12115-12120.

Sunagawa S, Coelho LP, Chaffron S, Kultima JR, Labadie K, Salazar G, Djahanschiri B, Zeller G, Mende DR, Alberti A, Cornejo-Castillo FM, Costea PI, Cruaud C, d'Ovidio F, Engelen S, Ferrera I, Gasol JM, Guidi L, Hildebrand F, Kokoszka F, Lepoivre C, Lima-Mendez G, Poulain J, Poulos BT, Royo-Llonch M, Sarmento H, Vieira-Silva S, Dimier C, Picheral M, Searson S, Kandels-Lewis S, Tara Oceans C, Bowler C, de Vargas C, Gorsky G, Grimsley N, Hingamp P, Iudicone D, Jaillon O, Not F, Ogata $\mathrm{H}$, Pesant S, Speich S, Stemmann L, Sullivan MB, Weissenbach J, Wincker P, Karsenti E, Raes J, Acinas SG, Bork P (2015) Structure and function of the global ocean microbiome. Science, 348,

\section{9.}

Theuns S, Vanmechelen B, Bernaert Q, Deboutte W, Vandenhole M, Beller L, Matthijnssens J, Maes P, Nauwynck HJ (2018) Nanopore sequencing as a revolutionary diagnostic tool for porcine viral enteric disease complexes identifies porcine kobuvirus as an important enteric virus. Scientific Reports, 8, 9830.

Tsai YC, Conlan S, Deming C, Segre JA, Kong HH, Korlach J, Oh J, Progra NCS (2016) Resolving the complexity of human skin metagenomes using single-molecule sequencing. mBio, 7, e01748-15.

Wick RR, Judd LM, Gorrie CL, Holt KE (2017) Completing bacterial genome assemblies with multiplex MinION sequencing. Microbial Genomics, 3, e000132.

Woese CR (1987) Bacterial evolution. Microbiological Reviews, 51, 221-271.

Woese CR, Fox GE (1977) Phylogenetic structure of the prokaryotic domain: The primary kingdoms. Proceedings of the National Academy of Sciences, USA, 74, 5088-5090.

Yang CY, Tarng DC (2018) Diet, gut microbiome and indoxyl sulphate in chronic kidney disease patients. Nephrology, 23, 16-20.

Youssef NH, Couger MB, Struchtemeyer CG, Liggenstoffer AS, Prade RA, Najar FZ, Atiyeh HK, Wilkins MR, Elshahed MS (2013) The genome of the anaerobic fungus Orpinomyces sp. strain C1A reveals the unique evolutionary history of a remarkable plant biomass degrader. Applied and Environmental Microbiology, 79, 4620-4634.

Zhao L, Song Y, Li L, Gan N, Brand JJ, Song L (2018) The highly heterogeneous methylated genomes and diverse restriction-modification systems of bloom-forming Microcystis. Harmful Algae, 75, 87-93.

(特邀责任编委: 周欣 责任编辑: 时意专) 